\title{
Efeitos antinociceptivos e sedativos da buprenorfina, da acepromazina ou da associação buprenorfina e acepromazina em gatos
}

\author{
Antinociceptive and sedative effects of buprenorphine, acepromazine, or combination of both, \\ in conscious cats
}

\section{Luciana Alvarez Santana ${ }^{I}$ Stelio Pacca Loureiro Luna ${ }^{I}$ Paulo Vinicius Mortensen Steagall ${ }^{\text {I }}$ Tatiana Henriques Ferreira ${ }^{\text {III * }}{\text { Polly } \text { Taylor }^{\text {IV }} \text { Mike Dixon }}^{\text {IV }}$}

\section{RESUMO}

$O$ efeito antinociceptivo da buprenorfina tem sido relatado em cães e gatos. No presente estudo, avaliou-se o limiar nociceptivo mecânico em felinos tratados com buprenorfina, acepromazina ou ambas associadas e foram comparados os efeitos antinociceptivos e sedativos da associação em relação ao uso isolado desses fármacos determinados pelo mesmo observador, por meio de analgesiômetro e da escala analógica visual dinâmica interativa (DIVAS), respectivamente. Os oito animais empregados no estudo foram previamente familiarizados com os procedimentos utilizados. Após quatro mensurações basais, foram administrados, por via intramuscular, $0,02 \mathrm{mg} \mathrm{kg}^{-1}$ de buprenorfina, 0,06mg $\mathrm{kg}^{-1}$ de acepromazina ou 0,01 $\mathrm{mg} \mathrm{kg}^{-1}$ de buprenorfina associada a $0,03 \mathrm{mg}^{\mathrm{kg}^{-1}}$ de acepromazina, em um estudo cego, com delineamento em quadrado latino $e$ tratamento semanal. Os efeitos antinociceptivos $e$ sedativos foram avaliados aos 15, 30, 45 minutos e uma, duas, três, quatro, seis, oito e 12 horas após a administração do tratamento. O limiar nociceptivo mecânico se elevou significativamente apenas no grupo tratado com a associação buprenorfina-acepromazina (entre 45 minutos e uma hora). Em relação à sedação, nos grupos tratados com acepromazina $e$ com a associação, os valores da DIVAS foram significativamente maiores, respectivamente, de 15 minutos até quatro horas e de 15 minutos até três horas pós-tratamento, não apresentando elevação desses valores com a buprenorfina. Concluiu-se que não foi possível verificar a superioridade da neuroleptoanalgesia em relação ao uso dos fármacos isoladamente.

Palavras-chave: acepromazina, analgesia, antinocicepção, buprenorfina, gato, neuroleptoanalgesia.

\section{ABSTRACT}

The antinociceptive effects of buprenorphine have been reported in dogs and cats. This study evaluated changes in the mechanical nociceptive threshold and the sedative effects of buprenorphine, acepromazine and its combination in cats, determined by the same observer using a nociceptive threshold testing device and DIVAS, respectively. Eight animals were previously conditioned to the procedures. After four baseline measurements, $0.02 \mathrm{mg} \mathrm{kg}^{-1}$ of buprenorphine, $0.06 \mathrm{mg} . \mathrm{kg}^{-1}$ of acepromazine, or $0.01 \mathrm{mg} \mathrm{kg}^{-1}$ of buprenorphine with $0.03 \mathrm{mg}$ $\mathrm{kg}^{-1}$ of acepromazine were administered intramuscularly in a blinded and experimental study using a Latin square design within a one week interval between treatments. The antinociceptive and sedative effects were evaluated at 15, 30, 45 minutes and $1,2,3,4,6,8$ and 12 hours post treatment. The nociceptive threshold increased significantly only after the combination buprenorphine-acepromazine (between 45 minutes and 1 hour). Regarding sedation, the use of acepromazine and the combination of both were associated with significantly higher DIVAS values from 15 minutes to 4 hours and 15 minutes to 3 hours post treatment, respectively. No increase in these values was noted with the use of buprenorphine. It was concluded that it could not be verified the superiority of neuroleptanalgesia over the use of drugs alone

Key words: acepromazine, analgesia, antinociception, buprenorphine, cat, neuroleptanalgesia.

\section{INTRODUÇÃO}

O emprego de analgésicos em gatos ainda é limitado devido ao medo dos efeitos colaterais dos

IDepartamento de Cirurgia e Anestesiologia Veterinária, Faculdade de Medicina Veterinária e Zootecnia (FMVZ), Universidade Estadual Paulista Júlio de Mesquita Filho (Unesp), Botucatu, SP, Brasil.

"Ontario Veterinary College - Health Sciences Centre, Guelph, Ontario, Canada.

IIIVeterinary Medical Teaching Hospital, School of Veterinary Medicine, University of California, Davis, One Shields Av, Davis, CA 95616, USA. E-mail: tathf@yahoo.com.br. *Autor para correspondência.

${ }^{\text {IV }}$ Taylor Monroe, Little Downham, Ely, UK 
analgésicos tradicionais, à falta de fármacos autorizados para o uso nessa espécie, aos poucos estudos a respeito da farmacocinética e da farmacodinâmica e ao fato de as doses dos medicamentos utilizados serem extrapoladas de avaliações realizadas em outras espécies de animais, especialmente a canina (ROBERTSON \& TAYLOR, 2004).

Os opioides são considerados agentes importantes devido a sua eficácia e segurança para o manejo da dor, sendo inclusive sua administração recomendada em pacientes críticos (ROBERTSON, 2005). Na espécie felina, o emprego de opioides ainda é restrito devido ao receio de excitação quando usados em doses elevadas (TAYLOR \& ROBERSTON, 2004; ROBERTSON, 2008). Porém, quando são conhecidas a farmacocinética e farmacodinâmica dos analgésicos e respeitadas as doses e os intervalos de administração para a espécie, é possível evitar esses efeitos adversos e se beneficiar da analgesia promovida pelos fármacos disponíveis (TAYLOR \& ROBERSTON, 2004).

A buprenorfina é considerada um fármaco adequado para o uso perioperatório em gatos com dor leve a moderada devido à sua eficácia, fácil administração, longa duração de ação e ausência de efeitos colaterais, quando utilizada em doses clínicas (ROBERTSON \& TAYLOR 2004; ROBERTSON, 2008). Apresenta longo período de latência (JOHNSON et al., 2007), o que exige administração precoce no período pré-operatório, quando se deseja a analgesia preemptiva (ROBERTSON \& TAYLOR, 2004).

Em estudos clínicos realizados em gatos, com doses não equipotentes, a buprenorfina produziu analgesia mais efetiva do que a da morfina (STANWAY et al., 2002), da meperidina (SLINGSBY et al., 1998), do cetoprofeno e da oximorfona (DOBBINS et al., 2002), porém inferior a do meloxicam e do carprofeno(GASSEL et al., 2005; MOLLENHOFF et al., 2005).

A neuroleptoanalgesia é um estado de depressão do sistema nervoso central e analgesia produzido pela associação de um agente neuroléptico a um opioide (BREARLEY, 1994), resultando em analgesia, sedação, manutenção da estabilidade autonômica, neurológica e cardiovascular (BISSONNETTE et al., 1999). Além disso, promove sedação mais profunda do que a soma dos efeitos de dois fármacos utilizados de forma isolada, com a vantagem de não incrementar os efeitos colaterais e de viabilizar o uso de doses menores dos fármacos utilizados. Os animais apresentam sonolência, porém permanecem responsivos a estímulos (BREARLEY, 1994; STEAGALL et al., 2006).
$\mathrm{O}$ agente neuroléptico mais utilizado na medicina veterinária em pequenos animais é a acepromazina, pertencente ao grupo dos fenotiazínicos (CORTOPASSI \& FANTONI, 2002). Tais agentes diminuem a ansiedade e são comumente empregados em associação com opioides para potencializar a analgesia (PASCOE, 1992) e a sedação (STEPIEN et al., 1995), além de reduzir os riscos de excitação (LAMONT, 2002). Em gatos, os efeitos são caracterizados por uma leve sedação, protrusão da terceira pálpebra e leve hipotensão(BREARLEY, 1994).

Este estudo objetivou avaliar o limiar nociceptivo mecânico em felinos tratados com buprenorfina, acepromazina ou ambas associadas e comparar os efeitos antinociceptivos e sedativos da associação em relação ao uso isolado desses fármacos, por meio, respectivamente, do analgesiômetro desenvolvido por DIXON et al. (2007) e de uma escala de sedação.

\section{MATERIAL E MÉTODOS}

Animais

Após aprovação pela Câmara de Ética em Experimentação Animal da Unidade, foram empregados oito gatos (um macho e sete fêmeas) hígidos, adultos, castrados, pesando $3,8 \pm 0,1$, provenientes do gatil experimental da disciplina de Anestesiologia Veterinária.

Todos os animais foram imunizados contra clamidiose, panleucopenia, calicivirose e rinotraqueíte felinas e tratados contra endo e ectoparasiticidas periodicamente. Antes do início da pesquisa, foram realizados hemogramas completos, exames bioquímico, renal e hepático de cada animal, e durante esta realizaram-se também exames físicos periódicos. Todos os animais possuíam laudo de PCR negativo para imunodeficiência felina e leucemia felina, emitido pelo Laboratório de Diagnóstico Molecular do IBB-UNESP/ Botucatu-SP. Todos os animais empregados no estudo foram previamente familiarizados com os procedimentos utilizados no mínimo um mês antes do experimento iniciar, por meio do posicionamento do bracelete e da realização de mensurações do limiar antinociceptivo mecânico.

Mensuração do limiar nociceptivo mecânico

O limiar nociceptivo mecânico, mensurado nos animais sem o uso de contenção física, foi determinado pela resposta à aplicação de um estímulo moderado e transitório, conforme desenvolvido por DIXON et al. (2007). Antes de cada estímulo, o observador (PVMS) assegurava-se de que o animal não estivesse dormindo, comendo ou brincando. 
O estímulo foi aplicado por um bracelete plástico de $5 \mathrm{~g}$ ao redor do antebraço do animal contendo três pinos, com 2,4mm de diâmetro, formando um triângulo de $10 \mathrm{~mm}$ de superfície chata, os quais foram pressionados contra a superfície craniolateral do membro, por meio de um manguito de pressão arterial neonatal modificado e inflado manualmente. $\mathrm{O}$ manguito foi conectado a uma torneira de três vias, acoplada a uma seringa de $30 \mathrm{~mL}$ e a um transdutor de pressão por uma linha arterial não complacente. $\mathrm{O}$ êmbolo da seringa foi empurrado manualmente $(1,5 \mathrm{~mL}$ segundo ${ }^{-1}$ ) pelo observador até que o animal apresentasse alguma reação, tal como puxar o membro torácico contra o corpo ou vocalizar. A pressão foi registrada por um voltímetro digital e imediatamente liberada após a resposta observada. Caso o animal não respondesse ao estímulo, um sistema de segurança para prevenir lesão da pele era acionado automaticamente quando a seringa estivesse totalmente vazia, numa pressão máxima de $850 \mathrm{mmHg}$, levando à liberação imediata da pressão no manguito. Antes da administração de qualquer fármaco, quatro mensurações foram realizadas com intervalo de 15 minutos entre cada uma, sendo a média delas considerada o limiar de pressão basal.

Avaliação da sedação e do comportamento

Imediatamente antes de cada mensuração dos limiares nociceptivos, a sedação foi avaliada por observação postural do animal e grau de responsividade e interação com o meio ambiente, por meio da Escala Analógica Visual Dinâmica Interativa (DIVAS), caracterizada por uma reta horizontal de $100 \mathrm{~mm}$ de comprimento. A extremidade esquerda dessa reta, correspondente ao " 0 ", representou ausência de sedação, e o outro extremo correspondente ao "100" representou a maior sedação possível. Alterações de comportamento oriundas dos tratamentos foram avaliadas subjetivamente, mediante a observação dos animais. Foi considerada euforia quando os seguintes comportamentos se apresentaram com frequência aumentada: "afofar" com os membros anteriores, se tornarem mais carinhosos (buscando atenção e carinho com o observador e outros pesquisadores envolvidos), deitar e rolar.

\section{Tratamento analgésico}

Após as quatro mensurações basais, foram administrados, em cada animal, por via intramuscular, $0,02 \mathrm{mg} \mathrm{kg}^{-1}$ de buprenorfina, ou $0,06 \mathrm{mg} \mathrm{kg}^{-1} \mathrm{de}$ acepromazina, ou $0,01 \mathrm{mg} \mathrm{kg}^{-1}$ de buprenorfina associada a $0,03 \mathrm{mg} \mathrm{kg}^{-1}$ de acepromazina, num estudo cego utilizando-se delineamento em quadrado latino com uma semana de intervalo entre tratamentos. Os volumes calculados de todos os fármacos foram adicionados de solução fisiológica $0,9 \%$ até $0,3 \mathrm{~mL}$ no total. O limiar nociceptivo mecânico foi mensurado aos 15, 30, 45 minutos e uma, duas, três, quatro, seis, oito e 12 horas após a administração do tratamento.

\section{Análise estatística}

Os dados foram analisados por ANOVA para medidas repetidas seguida do teste de Dunnet para comparações das alterações ao longo do tempo dentro do mesmo grupo. As diferenças entre os grupos foram comparados por ANOVA seguido do teste de Bonferroni. As diferenças foram consideradas significativas quando $\mathrm{P}<0,05$.

As comparações foram realizadas por meio das avaliações da média do limiar após cada tratamento com a média das mensurações basais antes do tratamento. $\mathrm{O}$ valor de referência foi considerado a média do limiar mecânico para cada gato durante o período das mensurações basais, portanto antes do tratamento.

\section{RESULTADOS}

O limiar nociceptivo mecânico se elevou significativamente em relação ao basal apenas no grupo tratado com a associação buprenorfina-acepromazina, entre 45 minutos $(\mathrm{P}<0,05)$ e uma hora $(\mathrm{P}<0,01)$ (Figura 1). Não houve diferença significativa no limiar mecânico quando os tratamentos foram comparados entre eles. Ressalta-se que houve considerável variação individual em todos os tratamentos, pois alguns animais apresentaram grande aumento em seu limiar quando comparados a outros.

Em relação ao DIVAS, no grupo tratado com buprenorfina, não houve diferença significativa em comparação às medidas basais (Figura 2). Já no grupo tratado com acepromazina os valores da DIVAS foram significativamente maiores de 15 minutos até quatro horas pós-tratamento $(\mathrm{P}<0,01)$, atingindo pontuação máxima (47) aos 45 minutos (Figura 2). Na associação buprenorfina-acepromazina, os valores da DIVAS foram significativamente maiores de 15 minutos até três horas $(\mathrm{P}<0,05)$, com a pontuação máxima (37) na primeira hora após o tratamento (Figura 2). Quando os grupos foram comparados entre si, os valores da DIVAS foram maiores com o uso de acepromazina e na associação dos fármacos em relação à buprenorfina de 15 minutos até três horas $(\mathrm{P}<0,05)$. Esses valores também foram significativamente maiores nos animais tratados com acepromazina em relação à associação aos 45 minutos pós-tratamento $(\mathrm{P}<0,05)$. 


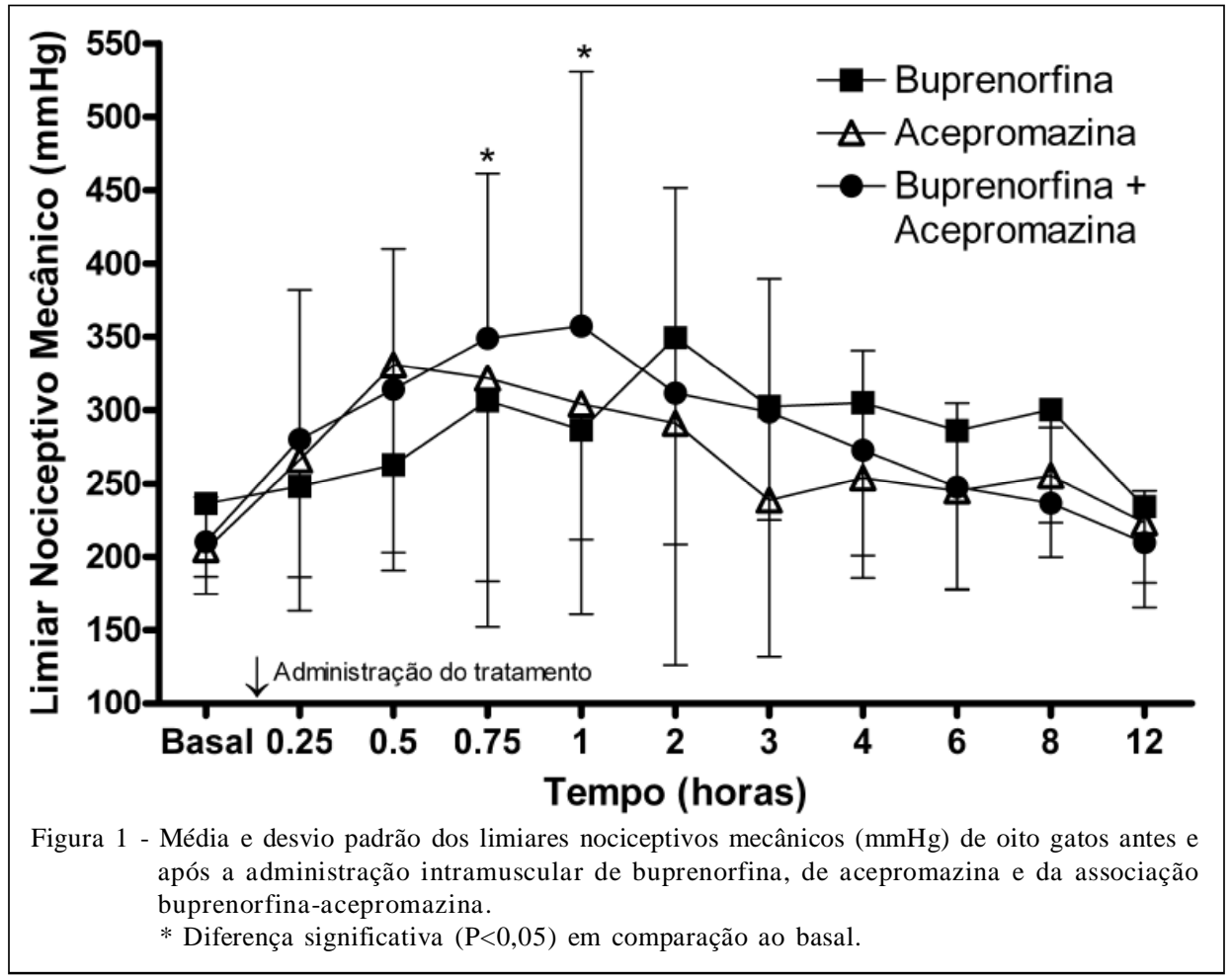

Considerando as alterações de comportamento avaliadas subjetivemente, todos os animais tratados com acepromazina e com a associação dos fármacos apresentaram sedação, principalmente entre 30 minutos e uma hora. Apesar de os animais tratados somente com a buprenorfina não apresentarem sedação, foram observadas alterações comportamentais compatíveis com euforia. Não foram observados disforia e êmese após a administração de nenhum tratamento.

\section{DISCUSSÃO}

$\mathrm{O}$ analgesiômetro utilizado no presente estudo foi validado e desenvolvido para detectar o limiar nociceptivo mecânico em gatos, representando um papel importante no estudo da analgesia nessa espécie (STEAGALL et al., 2007). No presente estudo, em nenhum caso, foi necessário o acionamento do sistema de segurança para prevenir lesão tecidual, visto que todos os animais responderam ao estímulo na pressão máxima de $401 \mathrm{mmHg}$. De acordo com os resultados de alguns estudos utilizando esse aparelho (STEAGALLet al., 2006; 2007; TAYLOR et al., 2007; STEAGALL et al., 2008; 2009), sugere-se que a elevação do limiar nociceptivo mecânico está associada com o efeito analgésico promovido pelo fármaco utilizado (STEAGALL et al., 2008).
Trabalhos empregando o analgesiômetro demonstraram efeito antinociceptivo com a utilização de diversos opioides, como a buprenorfina, a metadona, o butorfanol e a morfina; e outros agentes, como o tramadol e a acepromazina (STEAGALL et al., 2006; DIXONet al., 2007; STEAGALLet al., 2007; 2008; 2009). Entretanto, contrariando esses resultados, a administração isolada da buprenorfina e da acepromazina no presente estudo não promoveu elevação significativa do limiar nociceptivo mecânico. Esse fato pode estar relacionado com a alta variabilidade individual nas respostas aos agentes administrados nos animais deste estudo, que pode ter mascarado diferenças significativas nos efeitos analgésicos dos tratamentos utilizados. Essa variabilidade tem sido observada nessa espécie em resposta a diversos tratamentos analgésicos (ROBERTSON et al., 2003; JOHNSON et al., 2007; STEAGALL et al., 2008; 2009) e parece estar relacionada com fatores genéticos (ROBERTSON, 2008)

Esperava-se que a administração da acepromazina associada à buprenorfina potencializasse o efeito analgésico e sedativo da utilização isolada dos fármacos, como já foi observado em um estudo anterior com o uso de neuroleptoanalgesia, pelo menos quanto aos efeitos antinociceptivos (STEAGALL et al., 2008). Essa hipótese foi confirmada em relação aos efeitos 


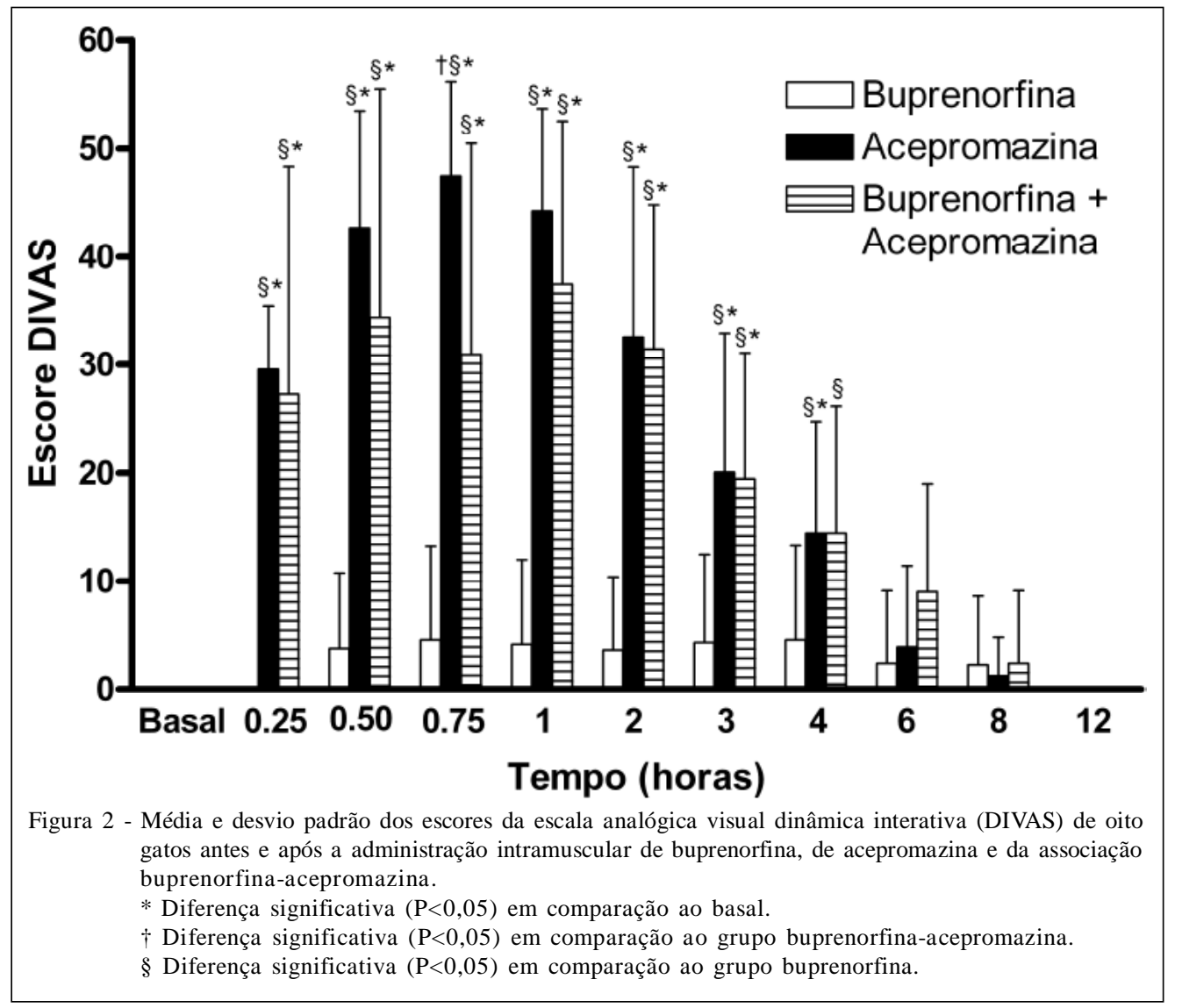

antinociceptivos, já que apenas a associação demonstrou resultados significativos. Porém, provavelmente devido à grande variabilidade dos limiares, a elevação significativa dos limiares nociceptivos com a utilização da associação foi extremamente curta (apenas $15 \mathrm{~min}$ ).

Quanto aos efeitos sedativos, os tempos de duração e valores de DIVAS foram semelhantes entre o grupo tratado com acepromazina e o tratado com a associação buprenorfina-acepromazina, portanto não se observou uma potencialização desse efeito com a utilização da associação. O efeito sedativo no grupo tratado com a associação pode ter sido mascarado pela euforia causada pela buprenorfina. Como esperado, o grupo tratado com buprenorfina não apresentou sedação, e sim euforia, o que é comumente relatado na literatura em gatos mediante a administração de doses adequadas de opioides (ROBERTSON \& TAYLOR, 2004; ROBERTSON, 2005; 2008).

Poderia-se suspeitar que essas alterações comportamentais e a sedação provocada pelos tratamentos utilizados poderiam comprometer a avaliação da resposta ao estímulo nociceptivo aplicado. Porém, apesar de o efeito sedativo da acepromazina poder reduzir a resposta nociceptiva dos animais mediante o estímulo aplicado, estudos anteriores utilizando metodologia semelhante já concluíram que, apesar de sedação profunda, os animais continuam a responder ao estímulo nociceptivo (SLINGSBY \& TAYLOR, 2008; STEAGALLet al., 2008).

Neste estudo optou-se por não utilizar um grupo controle com solução salina $0,9 \%$, já que diversos estudos demonstraram que não há alteração do limiar após a administração de solução salina $0,9 \%$ (STEAGALL et al., 2006; DIXON et al., 2007; STEAGALL et al., 2007; TAYLOR et al., 2007; STEAGALLet al., 2008).

\section{CONCLUSÃO}

Em razão da grande variabilidade individual e do curto efeito antinociceptivo significativo observado após a associação da buprenorfina e acepromazina, torna-se difícil a constatação da superioridade desse tratamento sobre o uso isolado dos fármacos. Além disso, os resultados observados em relação à sedação nos grupos acepromazina e associação acepromazina e buprenorfina foram 
significativos, porém semelhantes. Portanto, torna-se muito importante a avaliação individual dos animais e o uso de técnicas de farmacogenética para otimizar os efeitos dos fármacos.

\section{AGRADECIMENTO}

À Fundação de Amparo à Pesquisa do Estado de São Paulo (FAPESP), pela bolsa de iniciação científica do primeiro autor

\section{REFERÊNCIAS}

BISONNETTE, B. et al. Neuroleptoanesthesia: current status. Canadian Journal of Anesthesia, v.46, p.154-168, 1999. Disponível em: <http://springerlink.com/content/c133342387jwx247/ fulltext.pdf $>$. Acesso em: 12 jun. 2006.

BREARLEY, J.C. Sedation, premedication and analgesia. In: HALL, L.W.; TAYLOR, P.M. Anaesthesia of the cat London: Baillière Tindall, 1994. p.111-128.

CORTOPASSI, S.R.G.; FANTONI, D.T. Medicação préanestésica. In: FANTONI, D.T.; CORTOPASSI, S.R.G. Anestesia em cães e gatos. São Paulo: Roca, 2002. p.151158.

DIXON, M.J. et al. Development of a pressure nociceptive threshold testing device for evaluation of analgesics in cats. Research in Veterinary Science, v.82, p.85-92, 2007. Disponível em <http:/ /www.sciencedirect.com/science?_ob=ArticleURL\&_udi=B6WWR4 K5JBV6-1\&_user $=10 \& \_r d o c=1 \&$ fmt $=$ \&_orig $=$ search

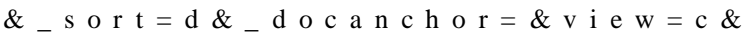
_acct $=\mathrm{C} 000050221 \&$ _version $=1 \&$ \&ur 1 Version $=0$ \&_userid=10\&md5=5a5a86a8531ce25bcafcc656ceb7061f $>$. Acesso em: 2 dez. 2007. doi: 10.1016/j.rvsc.2006.03.010.

DOBBINS, S. et al. Comparison of the effects of buprenorphine, oxymorphone hydrochloride, and ketoprofen for postoperative analgesia after onychectomy or onychectomy and sterilization in cats. Journal of the American Animal Hospital Association, v.38, p.507-514, 2002.

GASSEL, A.D. te al. Comparison of oral and subcutaneous administration of buprenorphine and meloxicam for preemptive analgesia in cats undergoing ovariohysterectomy. Journal of the American Animal Hospital Association, v.227, p. 19371944, 2005.

JOHNSON, J.A. et al. Antinociceptive effects of butorphanol, buprenorphine,or both, administered intramuscularly in cats. American Journal of Veterinary Research, v.68, p.699703, 2007. Disponível em: <http://avmajournals.avma.org/ doi/pdf/10.2460/ajvr.68.7.699>. Acesso em: 20 jul. 2008. doi: 10.2460/ajvr.68.7.699.

LAMONT, L.A. Feline perioperative pain management. Veterinary Clinics of North America: Small Animal Practice, v.32, p.747-763, 2002. Disponível em: <http:// w w w . science?_ob=ArticleURL\&_udi=B 758 P-47YYSPK$2 \&$ \& s e r $=10 \&$ \& c over D a t e $=07 \% 2 \mathrm{~F} 31 \% 2 \mathrm{~F}$ $2002 \&$ rdoc $=2 \&$ fmt $=$ high $\&$ _orig $=$ browse \&_srch $=$ docinfo( $\% 23$ toc\%2312932\%232002\%23999679995
$\% 23392837 \% 23$ FLA $\% 23$ display $\% 23$ Volume)\&_cdi $=1$ $2932 \&$ sort $=$ d\&_docanchor $=\&$ \&ct $=15 \&$ \& acct $=$ C000050221\& _ version $=1 \&$ \& ur 1 Version $=0 \&$ _ u seri d $=10 \&$ $\mathrm{md} 5=\mathrm{f} 829 \mathrm{a} 1 \mathrm{~b} 05 \mathrm{f} 3 \mathrm{bf} 59 \mathrm{bac} 4252 \mathrm{ee} 82891 \mathrm{aa} 2>$. Acesso em: 20 fev. 2003. doi: 10.1016/S0195-5616(02)00028-1.

MOLLENHOFF, A. et al. Anti-nociceptive efficacy of carprofen, levomethadone and buprenorphine for pain relief in cats following major orthopaedic sugery. Journal of Veterinary Medicine A., v.52, p.186-198, 2005.

PASCOE, P.J. The case for the routine use of analgesics. Veterinary Clinics of North America: Small Animal Practice, v.22, p.357-359, 1992.

ROBERTSON, S.A. Assessement and management of acute pain in cats. Journal of Veterinary Emergency and Critical Care, v.15, p.261-272, 2005

ROBERTSON, S.A. Managing pain in feline patients. Veterinary Clinics of North America: Small Animal Practice, v.38, p.1267-1790, 2008. Disponível em: <http:// w w w. s c i e n c e d i r e c t . c o m / science?_ob=MImg\&_imagekey=B 758P-4TT899J-9-

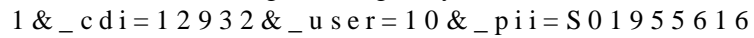
$0800137 \mathrm{X} \&$ orig=browse\&_coverDate $=11 \% 2 \mathrm{~F} 30$ $\% 2$ F2008\&_sk $=999619993 \& v i e w=c \& w c h p=d G L b V z W-$ zSkWb\&md5 $=4500 \mathrm{a} 89 \mathrm{ca} 32 \mathrm{a} 2 \mathrm{ebdd} 6 \mathrm{f} 392 \mathrm{cc} 4 \mathrm{ccf} 489 \mathrm{c} \& \mathrm{ie}=/$ sdarticle.pdf $>$. Acesso em: 30 out. 2008. doi: 10.1016/ j.cvsm.2008.06.008.

ROBERTSON, S.A.; TAYLOR, P.M. Pain management in cats past, present and future. Part 2. Treatment of pain - clinical pharmacology. Journal of Feline Medicine and Surgery, v.6, p.321-333, 2004. Disponível em: <http://www.sciencedirect.com/ science?_ob=MImg\&_imagek ey=B 6WJC-4B BVVMP-1 C\&_cdi $=6875 \&$ \& user $=10 \&$ \& pii $=$ S 1098612 X03001 $177 \&$ _orig $=$ browse $\&$ _coverDate $=10 \% 2$ F $31 \% 2$ F2004\&_sk $=999939994 \& \mathrm{view}=\mathrm{c} \& \mathrm{wchp}=\mathrm{dGLbVtz}-$ zSkWA\&md5=a35a4fcfae529f40494507a9f4 1ef732 \&ie $=$ / sdarticle.pdf> Acesso em: 31 dez. 2003. doi: 10.1016/ j.jfms.2003.10.002.

ROBERTSON, S.A. et al. Changes in thermal threshold response in eight cats after administration of buprenorphine, butorphanol and morphine. Veterinary Record, v.153, p.462-465, 2003.

SLINGSBY, L.S.; TAYLOR, P.M. Thermal antinociception after dexmedetomidine administration in cats: a dose-finding study. Journal of Veterinary Pharmacology and Therapeutics, v.31, p.135-142, 2008. Disponível em: <http:/ /onlinelibrary.wiley.com/doi/ $10.1111 /$ j. 1365 2885.2007.00931.x/pdf $>$. Acesso em: 12 fev. 2008. doi: 10.1111/j.1365-2885.2007.00931.x.

SLINGSBY, L.S. et al. Comparison of pethidine, buprenorphine and ketoprofen for postoperative analgesia after ovariohysterectomy in the cat. Veterinary Record, v.143, p.185-189, 1998.

STANWAY, G.W. et al. A preliminary investigation comparing pre-operative morphine and buprenorphine for postoperative analgesia and sedation in cats. Veterinary Anaesthesia and Analgesia, v.29, p.29-35, 2002. 
STEAGALL, P.V.M. et al. Effects of subcutaneous methadone, morphine, buprenorphine or saline on thermal and pressure thersholds in cats. Journal of Veterinary Pharmacology and Therapeutics, v.29, p.531-537, 2006. Disponível em: <http://onlinelibrary.wiley.com/doi/10.1111/j. 1365 2885.2006.00800.x/pdf $>$. Acesso em: 2 nov. 2006. doi: $10.1111 / \mathrm{j} .1365-2885.2006 .00800 . x$.

STEAGALL, P.V.M. et al. Effects of buprenorphine, carprofen and saline on thermal and mechanical nociceptive thresholds in cats. Veterinary Anesthesia and Analgesia, v.34, p.344350, 2007. Disponível em: <http://onlinelibrary.wiley.com/ doi/10.1111/j.1467-2995.2006.00331.x/pdf $>$. Acesso em: 8 maio, 2007. doi: 10.1111/j.1467-2995.2006.00331.x.

STEAGALL, P.V.M. et al. Antinociceptive effects of tramadol and acepromazine in cats. Journal of Feline Medicine and Surgery, v.10, p.24-31, 2008. Disponível em: <http:// www.sciencedirect.com/science?_ob=MImg\&_imagekey=B6WJC4PK8B B 9-1-1\&_cdi=6875\&_user $=10 \&$ \& pii $=$ S 1098 612 X07001313\&_orig=browse \&_coverDate= 02\%2F29\%2F2008\&_sk=999899998\&view $=c \& w c h p=d G L b V z W-$ zSkWb\&md5=3147618ea27aa6a67d9f19c243f62f43\&ie=/ sdarticle.pdf>. Acesso em: 4 set. 2007. doi: 10.1016/ j.jfms.2007.06.009.

STEAGALL, P.V.M. et al. Dose-related antinociceptive effects of intravenous buprenorphine in cats. Veterinary Journal, v.182, p.203-209, 2009. Disponível em: <http:// w w w. $\mathrm{s}$ c i e $\mathrm{n}$ c e d i r e c t. c o m / science?_ob=ArticleURL\&_udi=B6WXN-4T5JPJ6$3 \&$ _user $=687358 \&$ _coverDate $=11 \% 2$ F $30 \% 2$ F
$2009 \&$ \&doc $=1 \&$ \& $\mathrm{mt}=$ high\&_orig = search\&_origin= search $\&$ _sort $=d \&$ docanchor $=\&$ view $=c \&$ _searchStr $I d=1$ $480356815 \&$ \&rerunOrigin $=$ google $\&$ _acct $=$ C 000037899 \&_version $=1 \&$ _url Version $=0 \&$ _userid $=687358 \&$ $\operatorname{md} 5=e 1 \mathrm{f} 8 \mathrm{ca} 1 \mathrm{f} 359 \mathrm{fe} 4 \mathrm{f} 2 \mathrm{cbb} 894157 \mathrm{~b} 581 \mathrm{f} 25 \&$ searchtype $=\mathrm{a}>$. Acesso em: 8 ago. 2008. doi: 10.1016/j.tvj1.2008.06.017.

STEPIEN, R.L. et al. Cardiorespiratory effects of acepromazina maleate and buprenorphine hydrochloride in clinically normal dogs. American Journal of Veterinary Research, v.56, p.78-84, 1995.

TAYLOR, P.M.; ROBERTSON, S.A. Pain management in cats past, present and future. Part 1. The cat is unique. Journal of Feline Medicine and Surgery, v.6, 313-320, 2004. Disponível em: <http:/ /www.sciencedirect.com/science?_ob=MImg\&_imagekey=B6WJC-

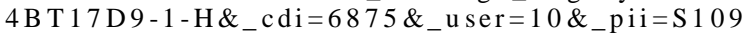
8612 X $03001189 \&$ o r i g = b r ow s e \&_c o $\mathrm{v}$ e r D a t e $=10 \% 2 \mathrm{~F} 31 \% 2 \%$ $99939994 \&$ vi e w $=\mathrm{c} \& \mathrm{w}$ c h p $=\mathrm{d} \mathrm{G} \mathrm{L} \mathrm{z} \mathrm{V} \mathrm{t} \mathrm{b} \mathrm{-}$ zSkzV\&md5=ca 19 f3ac3ec449ec3395e 5dd580a8282\&ie=/ sdarticle.pdf>. Acesso em: 26 fev. 2004. doi: 10.1016/ j.jfms.2003.10.003

TAYLOR, P.M. et al. Carprofen and buprenorphine prevent hyperalgesia in a model of inflammatory pain in cats. Research in Veterinary Science. v.83, p.369-375, 2007. Disponível em: <http:/ /www.sciencedirect.com/science?_ob=MImg\&_imagekey=B6WWR4NB 2SMS - $1-1 \&$ \& di $=7137 \&$ \& ser $=10 \&$ \&ii $=S 003452$ $\begin{array}{llllllllllllllllllll}8 & 8 & 0 & 7 & 0 & 0 & 0 & 1 & 2 & 4 & \&\end{array}$

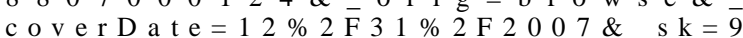
99169996 \& vi e w = c \& w c h p =d G L b V z W zSkzV\&md5=c4af6ccc3110afb $120 \mathrm{e} 29725 \mathrm{a} 01 \mathrm{dc} 68 \mathrm{~b} \& \mathrm{ie}=/$ sdarticle.pdf $>$. Acesso em: 23 mar. 2007. doi: 10.1016/ j.rvsc. 2007.01.007. 